\title{
Hybrid Mean Fuzzy Approach for Attention Detection
}

\author{
https://doi.org/10.3991/ijoe.v17i06.22315 \\ Haslinah Mohd Nasir ${ }^{(凶)}$, Mai Mariam Mohamed Aminuddin, \\ Noor Mohd Ariff Brahin, Mohd Syafiq Mispan \\ Universiti Teknikal Malaysia Melaka, Melaka, Malaysia \\ haslinah@utem.edu.my
}

\begin{abstract}
Statistics around the world showed that attention deficit significantly leads to road accidents. Hence, the growth of studies on attention deficit detection becoming more important. The studies obtained the waveform from electroencephalography (EEG) to identify the characteristic of attention. However, each individual has own unique characteristics to significantly shown the attention deficit. Thus, this research aim is to use the fuzzy approach to minimize the variability gap of the EEG signal between each individual. The research conducted the prior experiment to develop control parameter for training set of fuzzy by using two distinct stimulations to create two groups of attention sample i.e., attentive and inattentive. An approach of novel Hybrid Mean Fuzzy (HMF) was proposed in this research to detect attention deficit in EEG signal. It is the combination of simple averaging (Mean) and Fuzzy approaches for EEG analysis and classification. The results of using this method shows a significantly change in EEG signal which correlates to the attention detection. An Attention Degradation Scale (ADS) is successfully developed as the threshold value of EEG for attention detection. Therefore, the findings in this research can be a promising foundation on attention deficit detection in large application not only for reducing the road accidents.
\end{abstract}

Keywords - Biomedical signal processing, encephalography, simple averaging, fuzzy, hybrid intelligence system

\section{$1 \quad$ Introduction}

Attention is a basic part of cognitive system that able to reduce the uncertainty in the cognitive control in the function of orienting, alerting and executive control [1]. The information processing system performs each task in a serial order. To put in perspective, humans could still be able to perform two tasks simultaneously at the same time as long as the attention resource capacity is not exceeded. The illustration for easy understanding on how the attention works when perform two tasks at the same time is shown in Fig. 1. 


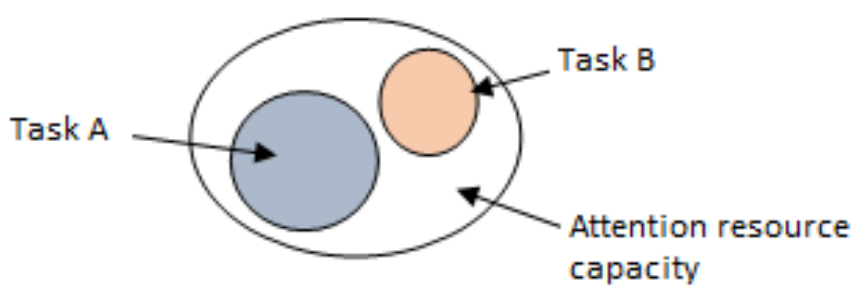

Fig. 1. The illustration if two tasks were done simultaneously

Attention is crucial to maintain the driver performance during driving. Attention loss during driving may lead to road accidents that may cause injuries and even fatalities. This situation is happening around the world and obviously it is a worldwide issue. The facts and statistics showed that attention loss during driving is a significant problem. Based on the World Health Organization (WHO) statistics, approximately 1.35 million people die each year as a result of a road traffic crash [2]. One of the risk factors of traffic crash is distracted driving which associates with the driver attention. Attention loss including drowsiness, sleepiness, as well as distracted driving may affects the driving performance. Road accidents may occur when the driver's ability to perception, recognition and vehicle control decrease during driving. It would be better if there is an effective method to detect whenever the attention is decline and warns the driver to reduce the road accidents. However, there are substantial challenges in detecting, measuring and addressing attention lose during driving. Thus, this paper proposed the use of encephalography (EEG) physiological measurement for attention detection. The choice of EEG is because it is proven to be fast tool for instant examination for attention evaluations [1,3-4].

Authors in [5] mentions that low perceptual demands may lead to mind shifted which may cause attention loss. This is supported by authors in [6] which revealed that the driving performances fluctuates when the attention loss during the lower perceptual load with longer driving duration.

There are several methods that been used for attention detection through EEG signal. Ref. [7] proposed K-Nearest Neighbors (KNN) algorithm to measure level of attention using BCI system. The authors managed to identify three levels of attention with some limitations. The classifier is unable to process when the EEG signal is too big.

While Ref. [8] proposed the temporal and time frequency features analysis to classify the attention during motor movement execution. Their findings revealed that the proposed method was able to predict the attention while performing a motor task. However, to analyze the effect of attention from the combination of motor movement and attention itself is impossible using this method.

In regards of the literature survey, attention detection based on EEG is a robust method as well as can be used effectively in various type of attention in combinations of few methods. This statement was supported in [9-12]. 
Artificial intelligence approach as classification is quite versatile for EEG signal estimation and prediction. This paper is basically use Fuzzy system approach for attention classification. It is known that fuzzy logic has an inference style that capable empowers human to apply reasoning in an artificial knowledge-based system [13]. Using fuzzy as classification approaches has garnered significant interest including for attention monitoring application.

Ref. [14] applied the fuzzy interference system to classify the distraction level from the amplitude of theta, alpha and beta of EEG waveform. The authors are able to classify the distraction into four different level; neutral, low, medium and high using the proposed approach.

The use of fuzzy logic approach was also proposed by Ref. [15] to classify the vigilance state based on the EEG signals. The authors revealed that the fuzzy logic has potential to classify the vigilance state into three different stages namely 'Awake; 'Slow Wave Sleep' and 'Rapid Eye Movement'.

Meanwhile, Ref. [16] findings have demonstrated that by using fuzzy classifier, the driver's drowsiness level can be differentiated into four levels specifically awake, drowsy, high drowsy and sleep.

In regards, the research based on fuzzy as attention classifier has become the benchmark for this paper. This paper will propose the combination of simple averaging (Mean) method and fuzzy system so called as Hybrid Mean Fuzzy (HMF) to predict the attention level specifically for driver during driving.

This paper is organized as follows. Section 2 describes on the proposed methodology for attention detection. In Section 3, the result and discussion are elaborated. Lastly, Section 4 will conclude and recommend improvement for future work.

\section{Methodology}

\subsection{Attention detection development}

The procedures involved in the attention detection development are the method used for attention detection as well as its hardware implementation. Basically HMF was used to develop the attention scale to detect the level of attention. The Mean method will be used in for data analysis and pre classification process while the Fuzzy approach will be utilized in data classification. In addition, the hardware implementation with the driver warning system was proposed. Fig. 2 shows the Flowchart of the whole system of attention detection. 


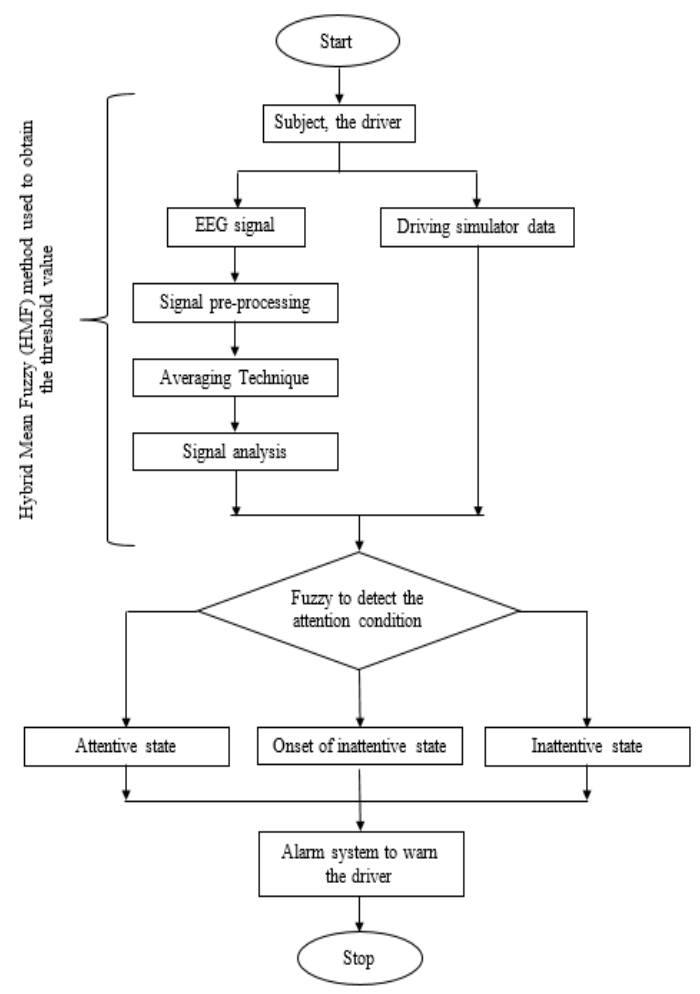

Fig. 2. The flowchart of attention detection procedure

\subsection{Data acquisition}

To acquire the EEG signal data, the setup is shown in Fig. 3 which consists of the human subject, verbal stimulant, bio signal amplifier, Carnersoft driving simulator and computer for data storing. The verbal stimulant is a radio channel selected by the subjects as a trigger.

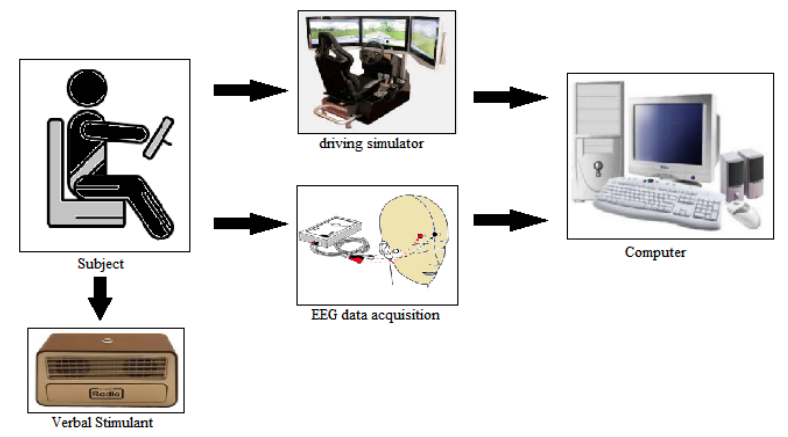

Fig. 3. The setup of the conducted process 
A total of 150 subjects who were normal healthy adults between the age of 18 and 30 years old (mean, $\mu$ is 23.18 and the standard deviation, $\sigma$ is 2.56) took part in the study on a voluntary basis. 100 subjects were required to drive the driving simulator with two different stimulations. The data from the total of the subjects was divided into two sets of data; training and testing data. The other 50 subjects become the control variable to justify the collected data. Table 1 shows the number of subjects that participated with their purpose.

Table 1. Number of subjects who participated

\begin{tabular}{|c|l|c|}
\hline Number of Subjects & \multicolumn{1}{|c|}{ Task } & Purpose \\
\hline 50 & $\begin{array}{l}\text { Driving with two different stimulations during driving; with and } \\
\text { without listening to radio }\end{array}$ & Training data \\
\hline 50 & $\begin{array}{l}\text { Driving with two different stimulations during driving; with and } \\
\text { without listening to radio }\end{array}$ & Testing data \\
\hline 50 & Watching video & Controlled data \\
\hline
\end{tabular}

The subjects were asked to drive the driving simulator in normal driving conditions throughout the recording to avoid any unwanted signals. For controlled data, the subjects were give two conditions to predict the attention level. The first 25 subjects experienced the same and repeated video to obtain the degrading pattern of EEG waveform while the other 25 subjects experience different interesting video to let the subject stay focused at whole time.

The procedural was conducted on high fidelity in lab driving simulator. The subjects were seated in front of the driving simulator while attached to the electrodes of EEG as shown in Fig. 4. Both driving and EEG data will be collected simultaneously.

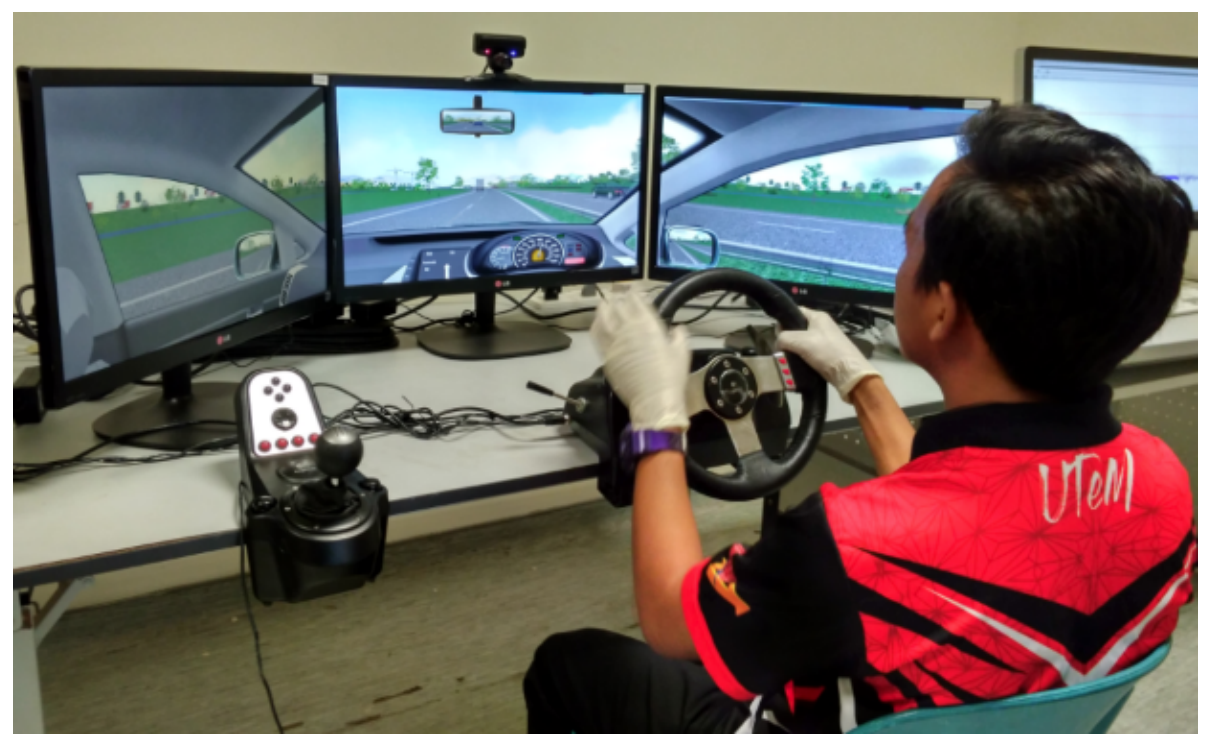

Fig. 4. The procedural of EEG data acquisition 
The subjects were given two different stimulations; with (up to $65 \mathrm{~dB}$ ) and without (up to $50 \mathrm{~dB}$ ) listening to the radio during the driving. Trigger sound of $1 \mathrm{kHz}$ tone was played throughout the procedure as a time-locked stimulus for both stimulations. The EEG data was collected using BIOPAC system which the impedance is guaranteed to be under $5 \mathrm{k} \Omega$ and the bandpass filter is between 4 to $30 \mathrm{~Hz}$ with sampling frequency $500 \mathrm{~Hz}$.

\subsection{Data acquisition}

Prior to analysis, pre-processing of EEG signal is crucial to remove all the artefacts. The BIOPAC system is set to $50 \mathrm{~Hz}$ notch filter to reduce the artifacts in the collected EEG data. In addition, removing artefacts using the visual inspection through MATLAB coding was done to finally produce the EEG signal with reduced artefacts for data analysis.

For data analysis, time domain simple averaging (Mean) approach was used as it is the simplest yet convincing compared to other methods [17]. The algorithm of MATLAB was developed to analyze the EEG signal. The averages for every 500 responses of window frames were extracted from the EEG signal where the mean from the amplitude of complex waveform of the time window containing the desired ERP component was computed. The time window is determined visually from the grand average which was computed by averaging at least 100 trials [18]. The procedure of the analyzing ERP wave of EEG signal using simple averaging method is shown as follows:

Let $M$ represent an average EEG dataset and $i$ is a trial

$$
M=i_{j} ;\{j=1,2, \ldots, N\}
$$

Where $N$ denotes the total of trials.

All successful trials were group and averaged. The illustration of mean averaging method for analysis is shown in Fig. 5. The three samples of averaged trials from ERP signal were evaluated. This cause the amplitude of ERP was reduced over the stimulation. According to Ref. [19], the assessment of the whole trial was obtained through the averaging process for each trial in every group. This approach also reduced the signal to noise ratio and it was able to demonstrate clear ERP wave amplitude accordingly. 


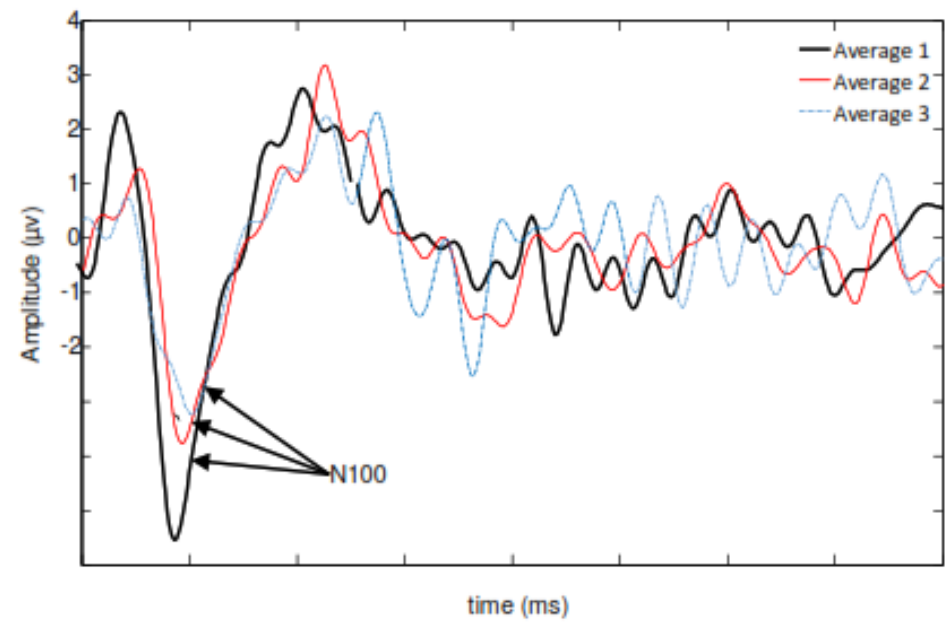

Fig. 5. Plotted EEG signal using simple averaging (mean) approach [20]

The extracted EEG signal from mean averaging approach will be then go through the fuzzy system, specifically fuzzy rule-based method. This is why the proposed method called as Hybrid Mean Fuzzy (HMF). According to Ref. [20], fuzzy logic provides an effective tool for extraction and measurement based on ambiguity and vagueness data and human perceptions. The algorithm of fuzzy was written in MATLAB. The inputs for fuzzy are EEG data (post analysis through mean averaging approach) and the accident score (the score of the driving performance based on the driving simulator grading). These inputs will generate the result of the attentiveness condition of the driver using the developed fuzzy algorithm. The block diagram of the fuzzy rule based for the system is shown in Fig. 6 while Table 2 explains the matrix of fuzzy.

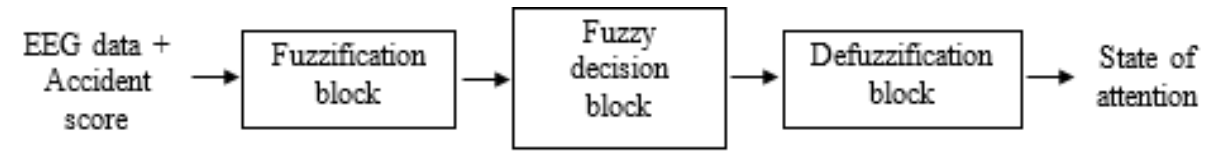

Fig. 6. Block diagram of Fuzzy rule based

Table 2. Fuzzy matrix inference system

\begin{tabular}{|c|l|l|l|l|}
\hline & \multicolumn{1}{|c|}{ LN } & \multicolumn{1}{c|}{ SN } & \multicolumn{1}{c|}{ SP } & \multicolumn{1}{c|}{ LP } \\
\hline L & Inattentive State & Onset of Inattentive State & Attentive State & Attentive State \\
\hline M & Inattentive State & Inattentive State & Onset of Inattentive State & Attentive State \\
\hline H & Inattentive State & Inattentive State & Inattentive State & Onset of Inattentive State \\
\hline
\end{tabular}

The rule define from the above matrix inference system are as follows: 
a) If the attention level from EEG data is large negative (LN) and the accident score is low (L) then the output is Inattentive State

b) If the attention level from EEG data is large negative (LN) and the accident score is medium (M) then the output is Inattentive State

c) If the attention level from EEG data is large negative (LN) and the accident score is high $(\mathrm{H})$ then the output is Inattentive State

d) If the attention level from EEG data is small negative (SN) and the accident score is low (L) then the output is the onset of Inattentive State

e) If the attention level from EEG data is small negative (SN) and the accident score is medium (M) then the output Inattentive State

f) If the attention level from EEG data is small negative (SN) and the accident score is high $(\mathrm{H})$ then the output is Inattentive State

g) If the attention level from EEG data is small positive (SP) and the accident score is low (L) then the output is Attentive State

h) If the attention level from EEG data is small positive (SP) and the accident score is medium (M) then the output is the onset of Inattentive State

i) If the attention level from EEG data is small positive (SP) and the accident score is high $(\mathrm{H})$ then the output is Inattentive State

j) If the attention level from EEG data is large positive (LP) and the accident score is low (L) then the output is Attentive State

k) If the attention level from EEG data is large positive (LP) and the accident score is medium (M) then the output is Attentive State

1) If the attention level from EEG data is large positive (LP) and the accident score is high $(\mathrm{H})$ then the output is the onset of Inattentive State

\section{Results and Discussion}

As for individual response, the data for one subject was selected randomly to show the EEG waveform and the accident score from the driving simulator grading for both stimulations. Fig. 7 and Fig. 8 show the EEG data and the accident score for driving without and with stimulation respectively. 


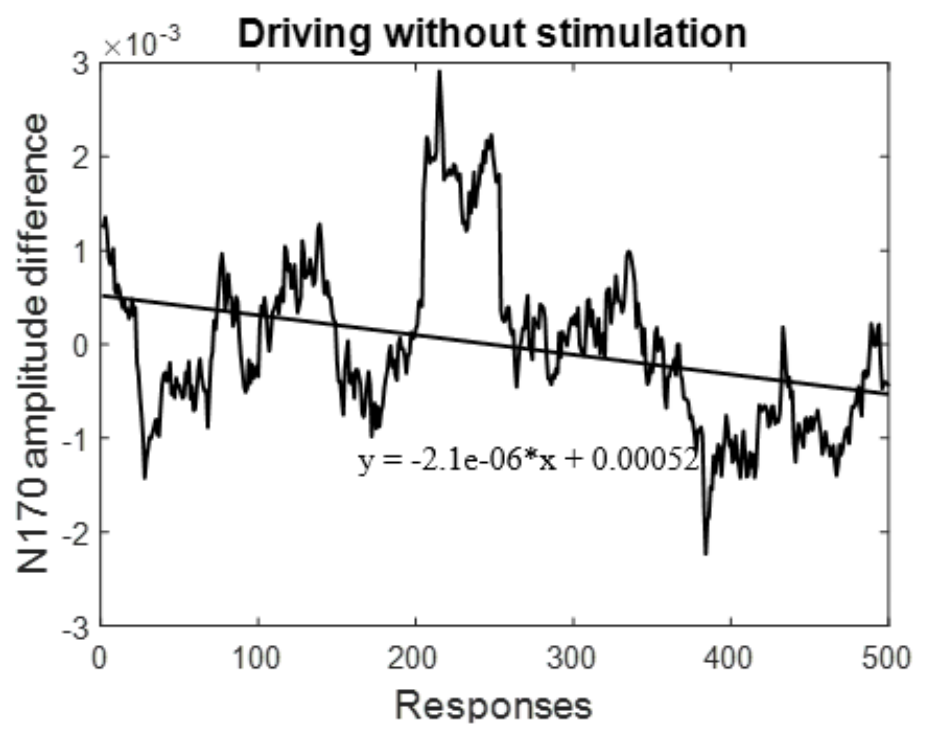

\begin{tabular}{|l|l|l|}
\hline Tasks & Grade & Main errors \\
\hline General & 5.00 & Accident \\
\hline
\end{tabular}

Fig. 7. Individual result of driving without stimulation

As can be seen from Fig. 7, it is apparent that the amplitude of ERP waveform of EEG is significantly declined over the time. In addition, the accident data from the driving grading shows the red colour and the grade is only 5 over 10 . This result indicates that the driver has failed the driving task. Both results show that the attention of the driver significantly degrading and the accident might happen. 


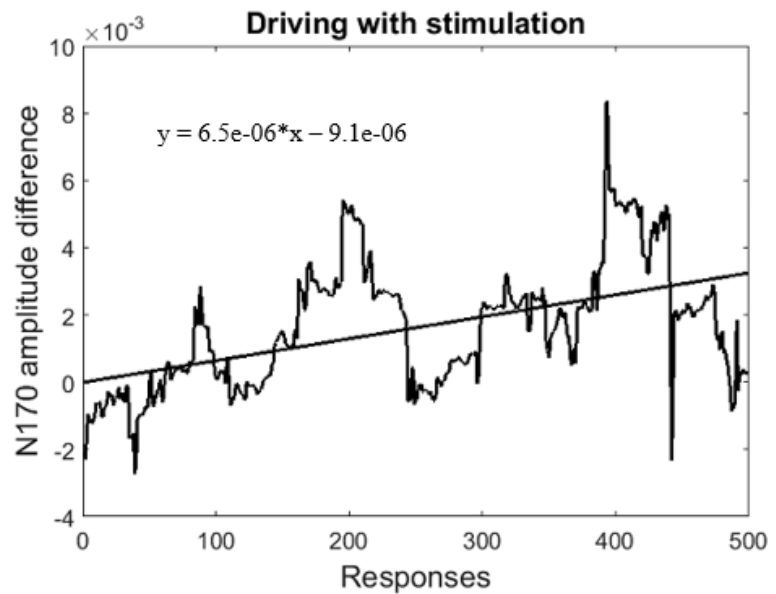

\begin{tabular}{|l|l|l|}
\hline Tasks & Grade & Main errors \\
\hline General & 9.00 & Route error \\
\hline
\end{tabular}

Fig. 8. Individual result of driving with stimulation

It is apparent from Fig. 8 that the ERP waveform of EEG signal reveals slight growth and the driving score show green color with grade of 9 over 10. The results show that the driver is still vigilant throughout the driving. From both Fig. 7 and Fig. 8 , it can be said the listening to radio (with stimulation) may help the driver to stay focus on the driving. The comparison from both Figures can be expressed in Table 3 .

Table 3. Data comparison of individual result

\begin{tabular}{|l|c|c|}
\hline & Without Stimulation & With Stimulation \\
\hline EEG data (Mean of ERP amplitude) & $-6.3441 \times 10^{-4}$ & $5.94 \times 10^{-4}$ \\
\hline Accident Score & 1 & 0 \\
\hline
\end{tabular}

From Table 3, it is predicted that an accident might occur when the driver's attention degraded (the mean of the ERP amplitude showed negative value) while when the driver was attentive (positive values of ERP amplitude), the road accident can be avoided. This finding is consistent with Ref. [19] where they discovered that the combination of submissive and dominant speech helps to increase the driver performance.

The grand average of 100 subjects (training and testing data) was analyzed to strengthen the individual result as shown above. Fig. 9(a) and Fig. 9(b) show the grand average without and with stimulation respectively. 


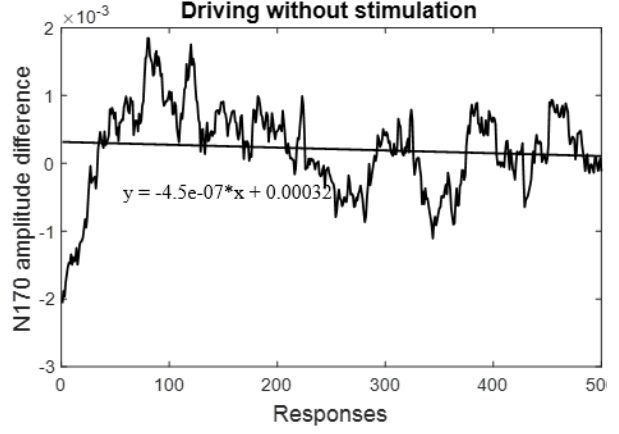

(a) Without stimulation

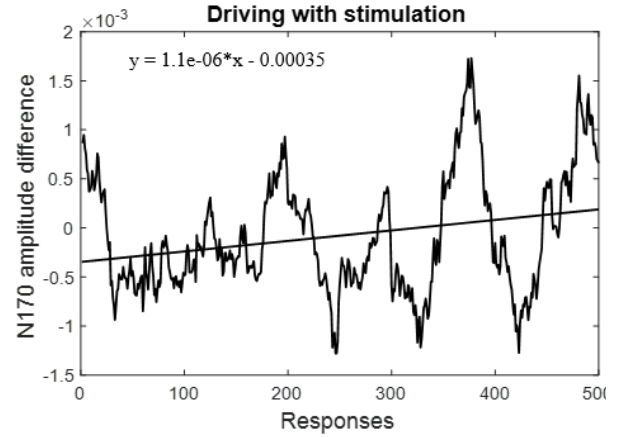

(b) With stimulation

Fig. 9. Grand average of 100 subjects

From Figure 9(a), apparently without stimulation the attention could easily degraded over the responses. While Fig. 9(b) shows a significant rise interpreted that the attention driver was in good condition. The morphology of the ERP waveform was taken into consideration at this part. Thus, Fuzzy method will be used at the next step for more accurate prediction. By using this HMF approach, the Attention Degradation Scale (ADS) was successfully developed as a threshold value of attention. To develop the ADS, the training data (50 out 100 subjects) were used. Fig. 10 shows the developed ADS in fish bone illustration.

\section{OUTPUT}
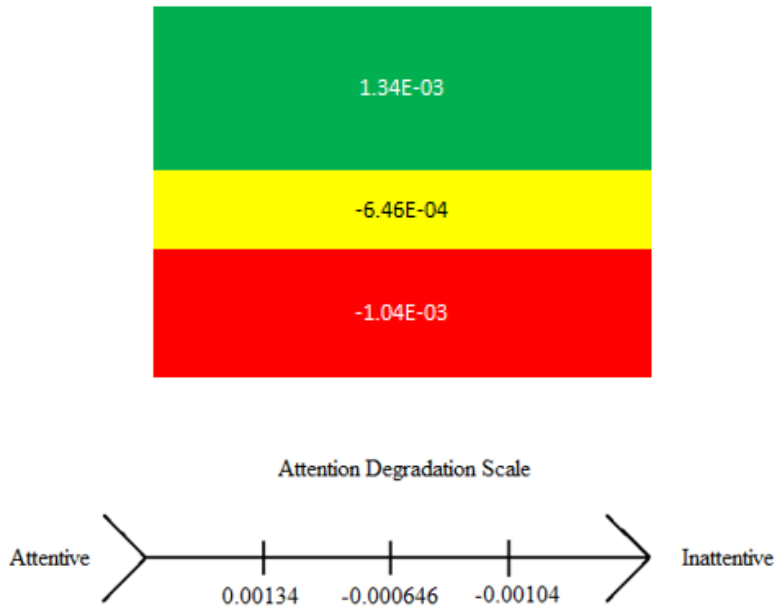

Fig. 10.Attention Degradation scale (ADS) developed from HMF approach 
The value towards the negative interprets that the mean different of ERP was decreased. It means that the subject attention is degraded which in inattentive state. In contrast, the value towards the positive reveals that the subject is attentive. While the middle value point out the onset of the inattentiveness. Based on the results gathered, $37.5 \%$ of the subjects were in attentive state, $53.1 \%$ were in the onset of inattentiveness and another $9.4 \%$ were in inattentive state.

Fuzzy algorithm is developed to detect the level of attention. In attentive state, the level is defined as ' 3 ', the onset of inattentiveness is defined as ' 2 ' while the inattentiveness state is stated as ' 1 '. The testing data were used for attention determination by utilizing the algorithm with and without the ADS. Fig. 11(a) and Fig. 11(b) are the output of Individual A and B from the testing data by using the algorithm.
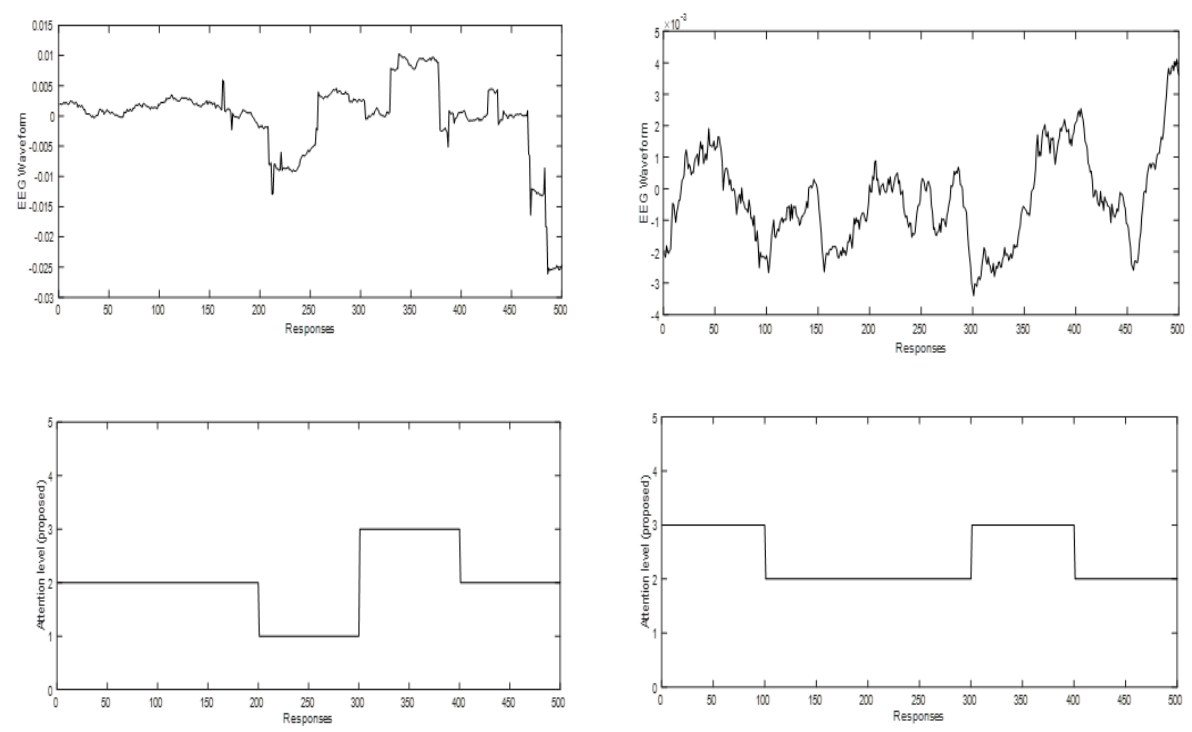

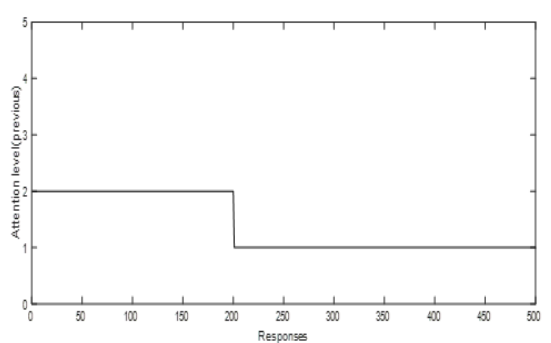

(a) Individual A

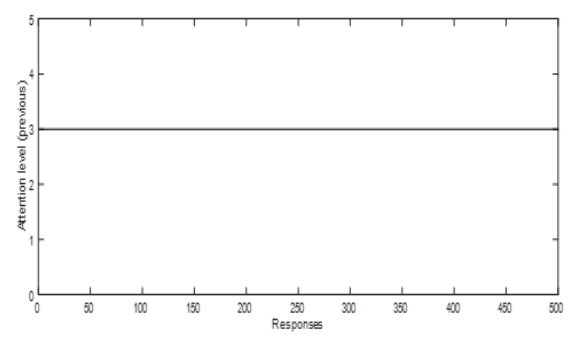

(b) Individual B

Fig. 11.With reference to EEG \& Attention levels using ADS 
According to Fig. 11(a) and Fig. 11(b), Picture number 1 is referred to as EEG waveform, picture number 2 is attention level using ADS while picture number 3 is referred to attention level without ADS. It is apparent from both figures that the level of attention is change accordingly to the EEG waveform. However, by using ADS, the attention is able to be detected compared to the one without ADS.

Referred to EEG waveform at Figure 11(b) for example, the attention should be decreased at response at 100 and increase back at responses of 300 . However it cannot be detected without ADS. By using ADS the changes is detected accordingly.

From 50 testing data, it was found that $52 \%$ of the subjects were detected as attentive while $48 \%$ were in inattentive state by using ADS. However, without using ADS the algorithm only able to detect $28 \%$ of attentive and $20 \%$ are inattentive from the same testing data. This apparently show that the develop ADS from HMF approach is more beneficial and able to detect attention much better.

\section{Conclusion}

The results presented indicate that with stimulation (listening to the radio) can reduce the attention loss during driving. In regards of that, the significant and insignificant attention degrading which reflects to their driving performance has been presented. The attention scale named as Attention Degrading Scale (ADS) has been developed by using Hybrid Mean Fuzzy (HMF) approach which able to detect three different attention state; attentive, onset of inattentive and inattentive state. In order to check the feasibility of ADS, the validation result with and without using ADS have been performed. And the results indicate that by utilized ADS the detection percentage is higher than without it. However, there are still some limitations to improve where it can be tested out with real time data.

\section{$5 \quad$ Acknowledgment}

This work was supported by Malaysia Ministry of Higher Education and Universiti Teknikal Malaysia Melaka (UTeM) under grant FRGS/1/2017/TK08/FKEKKCETRI/F00338. The authors would like to thank to the Universiti Teknikal Malaysia Melaka students for the participation in this research.

\section{References}

[1] Li, X., Hu, B., Dong, Q., Campbelt, W., Moore P. and Peng, H. (2011). EEG-based attention recognition. $6^{\text {th }}$ International Conference on Pervasive Computing and Applications, Oct 26-28 2011, Port Elizabeth, South Africa, pp 196-201. https://doi.org/10.1109/icpca. 2011.6106504

[2] WHO, "Road traffic injuries". Online: https://www.who.int/news-room/fact-sheets/detail/ road-traffic-injuries\#: : text $=$ Key $\% 20$ facts,result $\% 20$ of $\% 20$ road $\% 20$ traffic $\% 20$ crashes.\& text=Road $\% 20$ traffic $\% 20$ crashes $\% 20$ cost $\% 20$ most,pedestrians $\% 2 \mathrm{C} \% 20$ cyclists $\% 2 \mathrm{C} \% 20 \mathrm{a}$ nd\%20motorcyclists. [Accessed on 20 November 2020] 
[3] Ghassemi, F., Moradi, M. H., Tehrani-doost, M. and Abootalebi, V. (2009). Classification of sustained attention level based on morphological features of EEG's independent components. Proceedings of the ICME International Conference on Complex Medical Engineering, Apr 9-11 2009, Tempe, AZ, USA, pp 1-6. https://doi.org/10.1109/iccme.2009 .4906628

[4] Katona, J. and Kovari, A. (2015). EEG-based Computer Control Interface for BrainMachine Interaction. International Journal of Online and Biomedical Engineering, 11(6): 43-48. https://doi.org/10.3991/ijoe.v11i6.5119

[5] Geden, M. , Staicu A. M. and Feng, J. (2018). The impacts of perceptual load and driving duration on mind wandering in driving. Transportation Research Part F: Traffic Psychology and Behaviour, 57: 5-16. https://doi.org/10.1016/j.trf.2017.07.004

[6] Lin, C. T., Chuang, C. H., Kerrick, S., Mullen, T., Jung, T. P., Ko, L. W., Chen, S. A. J., King T. and McDowell, K. (2016). Mind wandering tends to occur under low perceptual demands during driving. Scientific Reports, Nature Publishing Group, 1-11. https://doi.org/10.1038/srep21353

[7] Li, Y., Li, X., Ratcliffe, M., Liu, L, Qi, Y. and Liu, Q. (2011). A real time EEG-based BCI system for attention. Proceedings of the 2011 International Workshop on Ubiquitous Affective Awareness and Intelligent Interaction. pp 33-39. https://doi.org/10.1145/2030 $\underline{092.2030099}$

[8] Aliakbaryhosseinabadi, S., Kamavuako, E. N., Jiang, N., Farina, D., and MrachaczKersting, N. (2017). Classification of EEG signals to identify variations in attention during motor task execution. Journal of Neuroscience Methods, 284:27-34. https://doi.org/10. 1016/j.jneumeth.2017.04.008

[9] Hamadicharef, B., Zhang, H., Guan, C., and Wang, C. (2009). Learning EEG-based Spectral-Spatial Patterns for Attention Level Measurement. IEEE International Symposium on Circuits and Systems, May 24-27 2009, Taipei, Taiwan. pp 1465-1468. https://doi.org/10. 1109/iscas.2009.5118043

[10] Krigolson, O. E., Williams, C. C., and Colino, F. L. (2017). Using Portable EEG to Assess Human Visual Attention Using Portable EEG to Assess Human Visual Attention. Augmented Cognition. Neurocognition and Machine Learning: 11th International Conference, pp 56-65. https://doi.org/10.1007/978-3-319-58628-1 5

[11] Das, N., Bertrand, A., and Francart, T. (2018). EEG-based Auditory Attention Detection: Boundary Conditions for Background Noise and Speaker Positions. Journal of Neural Engineering, 15:6 1-18. https://doi.org/10.1101/312827

[12] Ribeiro, D., Teixeira, C. and Cardoso, A. (2018). Web-based Platform for Training in Biomedical Signal Processing and Classification: the Particular Case of EEG-based Drowsiness Detection. International Journal of Online and Biomedical Engineering, 14(3): 164171. https://doi.org/10.3991/ijoe.v14i03.8193

[13] El_Masahde, M. B. (2020). Fuzzy Logic Controller of New Strategy of Biomedical Measurements. International Journal of Online and Biomedical Engineering, 16(10): 133-150

[14] Wali, M. K., Murugappan, M., and Ahmad, B. (2013). Subtractive Fuzzy Classifier Based Driver Distraction Levels Classification Using EEG. Journal of Physical Therapy and Science, 25: 1055-1058. https://doi.org/10.1589/jpts.25.1055

[15] Nagpal, C. and Upadhyay, P. K. (2015). Sleep EEG Classification Using Fuzzy Logic. International Journal of Recent Development in Engineering and Technology, 4:1 6-12

[16] Murugappan, M., Wali, M. K., Ahmmad, R. B. and Murugappan, S. (2013). Subtractive fuzzy classifier-based driver drowsiness levels classification using EEG. International Conference on Communication and Signal Processing, ICCSP 2013 - Proceedings, Apr 35 2013, Melmaruvathur, India. pp 159-164. https://doi.org/10.1109/iccsp.2013.6577036

[17] Burns, M. D., Bigdely-Shamlo, N., Smith, N. J., Kreutz-Delgado, K., and Makeig, S. (2013). Comparison of averaging and regression techniques for estimating Event Related 
Potentials. Conference proceedings: Annual International Conference of the IEEE Engineering in Medicine and Biology Society. pp. 1680-1683. https://doi.org/10.1109/emb c. 2013.6609841

[18] Valente, A., Bürki, A., Laganaro, M., Frishkoff, G. A., and Graves, W. W. (2014). ERP correlates of word production predictors in picture naming: a trial by trial multiple regression analysis from stimulus onset to response. Frontiers in Neuroscience, 8:390 113. https://doi.org/10.3389/fnins.2014.00390

[19] Aminuddin, M. and Mustaffa, I. (2013). The effect of sound levels on attention deficit. 2013 3rd International Conference on Instrumentation, Communications, Information Technology and Biomedical Engineering (ICICI-BME), Nov 7-8 2013, Bandung, Indonesia. pp 131-134. https://doi.org/10.1109/icici-bme.2013.6698479

[20] Chandna R. and Ram, M. (2015). Reliability measures measurement under rule-based fuzzy logic technique. International Journal of Engineering Transactions A: Basics, 28:10 1486-1492. https://doi.org/10.5829/idosi.ije.2015.28.10a.12

\section{Authors}

Haslinah Mohd Nasir received her Bachelor Degree in Electrical - Electronic Engineering (2008) from Universiti Teknologi Malaysia (UTM), MSc (2016) and PhD (2019) in Electronic Engineering from Universiti Teknikal Malaysia Melaka (UTeM). She had 5 years (2008-1013) experience working in industry and currently a lecturer in UTeM. Her research interest includes microelectronics, artificial intelligence and biomedical.

Mai Mariam Mohamed Aminuddin is a senior lecturer at Universiti Teknikal Malaysia Melaysia (UTeM) and has been worked as educator for more than 18 years. She obtained TRIZ Certificated Instructorship Level 1 from MyTRIZ in 2018. She also is a certified TRIZ Practitioner Level 3 awarded by the International TRIZ Association (MATRIZ) in 2017. Her area of expertise is artificial intelligence, biomedical engineering and TRIZ.

Noor Mohd Ariff Brahin received B. Eng Electrical - Electronic (Hons) from Univeristi Teknologi Malaysia (UTM) in 2008. He had experienced working in semiconductor industries as a structural design engineer from 2008 until 2013. He is currently a teaching engineer in Universiti Teknikal Malaysia Melaka. His research interests include artificial intelligence, microelectronics and IC design.

Mohd Syafiq Mispan received B. Eng Electrical (Electronics) and M. Eng Electrical (Computer and Microelectronic System) from Universiti Teknologi Malaysia, Malaysia in 2007 and 2010 respectively. He had experienced working in semiconductor industries from 2007 until 2014 before pursu- ing his Ph.D. degree. He obtained his Ph.D. degree in Electronics and Electrical Engineering from University of Southampton, United Kingdom in 2018. He is currently a senior lecturer in Fakulti Teknologi Kejuruteraan Elektrik dan Elektronik, Universiti Teknikal Malaysia Melaka. His current research interests include hardware security, CMOS reliability, VLSI design, and Electronic Systems Design.

Article submitted 2021-02-25. Resubmitted 2021-03-23. Final acceptance 2021-03-25. Final version published as submitted by the authors. 